\title{
An Ultra-High Field Magnetic Resonance Spectroscopy Study of Post Exercise Lactate, Glutamate and Glutamine Change in the Human Brain
}

\author{
Andrea Dennis ${ }^{1 * t}$, Adam G. Thomas ${ }^{1,2+}$, Nancy B. Rawlings ${ }^{1}$, Jamie Near ${ }^{1,3}$, \\ Thomas E. Nichols ${ }^{1,4}$, Stuart Clare ${ }^{1}$, Heidi Johansen-Berg ${ }^{1}$ and Charlotte J. Stagg ${ }^{1,5}$ \\ ${ }^{1}$ Oxford Centre for Functional MRI of the Brain (FMRIB), Nuffield Department of Clinical Neurosciences, University of Oxford, \\ Oxford, UK, ${ }^{2}$ Section on Functional Imaging Methods, National Institute of Mental Health, National Institutes of Health, \\ Department of Health and Human Services, Bethesda, MD, USA, ${ }^{3}$ Douglas Mental Health University Institute and \\ Department of Psychiatry, McGill University, Montreal, QC, Canada, ${ }^{4}$ Department of Statistics and Warwick Manufacturing \\ Group, University of Warwick, Coventry, UK, ${ }^{5}$ Physiological Neuroimaging Group, Oxford Centre for Human Brain Activity \\ (OHBA), University of Oxford, Oxford, UK
}

\section{OPEN ACCESS}

Edited by:

J. A. Taylor,

Harvard University, USA

Reviewed by:

Can Ozan Tan,

Harvard Medical School, USA

Alexander Peter Lin,

Brigham and Women's Hospital, USA

*Correspondence:

Andrea Dennis

andrea.dennis@ndcn.ox.ac.uk

${ }^{\dagger}$ These authors have contributed equally to this work.

Specialty section:

This article was submitted to

Exercise Physiology,

a section of the journal

Frontiers in Physiology

Received: 01 June 2015 Accepted: 09 November 2015 Published: 17 December 2015

Citation:

Dennis A, Thomas AG, Rawlings NB, Near J, Nichols TE, Clare S, Johansen-Berg $\mathrm{H}$ and Stagg $\mathrm{CJ}$ (2015) An Ultra-High Field Magnetic Resonance Spectroscopy Study of Post Exercise Lactate, Glutamate and

Glutamine Change in the Human

Brain. Front. Physiol. 6:351.

doi: 10.3389/fphys.2015.00351
During strenuous exercise there is a progressive increase in lactate uptake and metabolism into the brain as workload and plasma lactate levels increase. Although it is now widely accepted that the brain can metabolize lactate, few studies have directly measured brain lactate following vigorous exercise. Here, we used ultra-high field magnetic resonance spectroscopy of the brain to obtain static measures of brain lactate, as well as brain glutamate and glutamine after vigorous exercise. The aims of our experiment were to (a) track the changes in brain lactate following recovery from exercise, and (b) to simultaneously measure the signals from brain glutamate and glutamine. The results of our experiment showed that vigorous exercise resulted in a significant increase in brain lactate. Furthermore, both glutamate and glutamine were successfully resolved, and as expected, although contrary to some previous reports, we did not observe any significant change in either amino acid after exercise. We did however observe a negative correlation between glutamate and a measure of fitness. These results support the hypothesis that peripherally derived lactate is taken up by the brain when available. Our data additionally highlight the potential of ultra-high field MRS as a non-invasive way of measuring multiple brain metabolite changes with exercise.

Keywords: brain, lactate, glutamate, magnetic resonance spectroscopy, exercise

\section{INTRODUCTION}

In the non-activated state, the brain's energy needs are met primarily by glucose. However, the brain has been shown to be capable of using substrates other than glucose when they are available to support activity, including lactate (Schurr et al., 1997; Bouzier-Sore et al., 2003; Overgaard et al., 2012; Schurr, 2014), pyruvate (Cruz et al., 2001; Sharma et al., 2003), and ketone bodies (Nybo et al., 2003; Chowdhury et al., 2014). During recovery from exercise, lactate is perhaps the most important of these additional substrates (Dalsgaard et al., 2002) as during high intensity exercise there is an increase in blood and brain lactate (Quistorff et al., 2008; van Hall et al., 2009). It has even been suggested that lactate may be preferred to glucose, possibly 'sparing' brain glucose metabolism during exercise (Quistorff et al., 2008; van Hall, 2010; Schurr, 2014). 
There is, therefore, a great deal of interest in the metabolic fate of peripherally derived lactate within the human brain as evidence emerges as to its role as a putative neuronal energy source (Pellerin and Magistretti, 1994; Bouzier-Sore et al., 2003; van Hall et al., 2009) and as a signaling molecule in neuronal plasticity (Yang et al., 2014). Whilst persistently elevated brain lactate may indicate pathology, transient increases are normal consequences of the energetic processes involved in vigorous exercise and are now also believed to be complimentary to healthy brain processes (Schurr, 2008; Dienel, 2012) and brain recovery (Bouzat et al., 2014; Brooks and Martin, 2015; Glenn et al., 2015).

It is now widely accepted that on-going cerebral activity results in the generation of lactate in the brain, with lactate produced within the astrocytes fuelling neuronal activities (Fox et al., 1988; Schurr et al., 1988; Pellerin and Magistretti, 1994) probably via the astrocyte-neuron lactate shuttle (ANLS) (Magistretti and Pellerin, 1999; Pellerin and Magistretti, 2012). Here, however, we are primarily interested in the fate of peripherally derived lactate generated during exercise. Lactate is both taken up and produced in the brain by reversible reactions (Hertz et al., 2014). In one direction, pyruvate is reduced to lactate while $\mathrm{NADH}$ is oxidized to NAD+. In the other direction, lactate is oxidized to pyruvate, and NAD+ is reduced to NADH. In this direction, pyruvate is hypothesized to be either fully oxidized, producing $6 \mathrm{CO}_{2}$ and $6 \mathrm{H}_{2} \mathrm{O}$, or partially oxidized, to allow for carbon atoms to participate in exchange reactions (Cerdán et al., 2006; Massucci et al., 2013) resulting in de novo synthesis of amino acid neurotransmitters, particularly glutamate (Hertz and Dienel, 2004). It is therefore possible for the pyruvate derived from lactate to be both oxidized to fuel neuronal activity and act as a metabolic intermediate in exchange reactions, such as those involved in glutamate recycling.

Glutamate is a non-essential amino acid and is the main excitatory neurotransmitter of the cerebral cortex. It does not cross the blood-brain barrier and is therefore synthesized within the brain from glucose and a variety of other precursors such as lactate (Hertz and Fillenz, 1999). In vivo studies have established that the glutamate-glutamine cycle between glutamatergic neurons and glia is a major metabolic flux, reflecting synaptic glutamate release (Shen and Rothman, 2002). The glutamate-glutamine cycling flux is directly coupled to neuroenergetics (Sibson et al., 1998) and thus they are both important cerebral metabolites. The ability to measure glutamate and glutamine therefore will greatly increase our understanding of brain metabolic processes and aid in our understanding of brain lactate metabolism pathways.

Although there have been a number of studies measuring brain lactate changes following exercise (Ide et al., 2000; Dalsgaard et al., 2004; van Hall et al., 2009; Volianitis et al., 2011) these often involve invasive measures of venous blood from the dominant jugular vein and technology restricted to examining metabolites only present in blood leaving the brain. Proton Magnetic Resonance Spectroscopy ( $\left.\mathrm{H}^{1} \mathrm{MRS}\right)$ offers an attractive alternative to directly measuring cerebral blood measures as it is able to characterize brain tissues in terms of the relative concentration of many brain metabolites, including lactate, glutamate, and glutamine. $7 \mathrm{~T}^{1} \mathrm{H}$ MRS is non-invasive, nonionizing, and has been widely used for human studies. Alternative methods exist which are capable of measuring changes in brain metabolites, but they have significant limitations. Positron Emission Tomography (PET) is able to quantify neurochemicals and their binding, but relies on ionizing radiation. ${ }^{13} \mathrm{C}$ MRS offers an alternative approach, where signal from ${ }^{13} \mathrm{C}$ can be recorded, either via an infusion of ${ }^{13} \mathrm{C}$ MRS or using hyperpolarization. However, although these techniques are perhaps the gold standard for this approach they involve both lengthy and expensive infusions or are still largely in development, neither of which would be appropriate to investigate the question here.

To date, only two studies have used ${ }^{1} \mathrm{H}$ MRS to investigate changes in brain metabolites with exercise (Dalsgaard et al., 2004; Maddock et al., 2011). Whilst Dalsgaard and colleagues (Dalsgaard et al., 2004) were unable to resolve to lactate peak, using similar methods Maddock et al. (2011) used MRS at $1.5 \mathrm{~T}$ to examine the change in lactate and a combined measure of the glutamate and glutamine (Glx) concentration following vigorous exercise. The authors reported a sustained increase in brain lactate following exercise together with a transient increase in brain Glx, suggesting that at least some of the lactate is only partially oxidized. Although these results provide important direct evidence for brain metabolite changes post-exercise in humans, there are some limitations to the study. For example, at lower field strengths, detection of the lactate signal is difficult (Dalsgaard et al., 2004), and it is not possible to distinguish between glutamate and glutamine, making it impossible to know which is driving any observed changes in the composite measure Glx.

The aim of this study was two-fold: to replicate the experiment conducted by Maddock et al. (2011) in a study of exerciseinduced brain lactate uptake and to further examine the change in brain glutamate in the presence of increased lactate as a proof of principle for in vivo post exercise multi-metabolite measurements. Here we used MRS at 7T to measure the relative levels of lactate, glutamate, and glutamine before and after exercise in 11 volunteers. We hypothesized that exercise would lead to global cerebral lactate uptake which would be fully metabolized during recovery. We expected to be able to resolve the signal from glutamine and glutamate but did not expect any significant changes in other measureable metabolites.

\section{MATERIALS AND METHODS}

\section{Participants}

Eleven healthy, untrained, adult volunteers [mean age 30.0 years (range 22-41), 4 male] participated. The study was conducted in accordance with the Declaration of Helsinki and was approved by the Central Office for Research Ethics Committee [Oxford REC B (10/H0605/48)]. All participants gave their written informed consent prior to participation.

\section{Experimental Design}

Each subject underwent two experimental sessions: an exercise session and a control session. The order of the sessions was counterbalanced across the group. Sessions were conducted 
at the same time of day within subject. The experimental protocol for each session consisted of $20 \mathrm{~min}$ of baseline (pre) MRS measurements, after which subjects were removed from the scanner for the 15-min exercise or control intervention. They then returned to the scanner for $40 \mathrm{~min}$ of postintervention (post) MRS measurement (Figure 1). During scanning, participants were instructed to lie awake but were not engaged in any task. Participants were asked to refrain from eating, drinking, or consuming caffeine in the $2 \mathrm{~h}$ prior to each session and to refrain from performing exhaustive exercise in the preceding $24 \mathrm{~h}$.

In the exercise condition, subjects were required to perform a discontinuous, graded exercise paradigm to $85 \%$ of their predicted maximum heart rate $\left(\mathrm{HR} ; \mathrm{HR}_{\max }\right)(208-0.7 \times$ age, $\pm 3 \%$ ) on a cycle ergometer (Monarch, UK). This protocol was selected to be comparable with the previous MRS/exercise study in the literature (Maddock et al., 2011), to allow for blood lactate to be rapidly produced endogenously without inducing severe fatigue that would make it difficult for participants to lie still in the MRI scanner, and without resulting in dehydration that might affect brain MRS measurements. Exercise began at $60 \mathrm{~W}$ for females and $90 \mathrm{~W}$ for males, and increased by $30 \mathrm{~W}$ every 3 min until $85 \% \mathrm{HR}_{\max }$ was reached. $\mathrm{HR}$ was measured using a Polar heart rate monitor (Polar Electro 20, Finland) and was recorded in the last $30 \mathrm{~s}$ of every $3 \mathrm{~min}$ stage. The mean length of this intervention was $14 \pm 3 \mathrm{~min}$. In the control condition, participants sat in a quiet seating area where they were provided with some light reading material for $15 \mathrm{~min}$.

A proxy measure of aerobic fitness, the Physical Work Capacity (PWC), was established post-hoc for each individual in line with the PWC at 150 beats per minute test (PWC150; Campbell et al., 2001). Work rate achieved in the final step of the exercise protocol was normalized by body weight to give an estimate of $\mathrm{PWC}$, measured in $\mathrm{W} \mathrm{kg}^{-1}$ at $85 \%$ maximal heart rate.

In both sessions, blood lactate was measured from a capillary sample from the big toe before the pre scans, at the end of the intervention session, and every 10 min post intervention. Blood lactate was measured using the Lactate-Pro electrochemical test strip (Lactate Pro, Arkray Japan). The toe was used as a measurement site as access to participants' fingers was impossible when the participant was inside the 7T Magnetom scanner bore. Toe measurements were selected during exercise in order to match measurement site with those acquired in between MRS acquisitions. Measurements from the toe have been reported in the literature (Forsyth and Farrally, 2000; Garland and Atkinson, 2008) as a practical alternative site during exercise testing.

On reaching the end of the exercise test, participants were not afforded any time for active recovery. Continuing exercise at a lower intensity (active recovery) increases muscular blood flow (Bangsbo et al., 1994) facilitating the elimination of lactate. Instead, to maximize circulating lactate, subjects were returned to the scanner as soon as possible where they lay still for the duration of the post-exercise scans.

\section{Magnetic Resonance Data Acquisition}

Magnetic resonance (MR) data were acquired at the University of Oxford using a $7 \mathrm{~T}$ MRI scanner (Siemens Magnetom $7 \mathrm{~T}$, Erlangen, Germany) with a 32-channel head coil (Nova Medical, MA, USA). Prior to MRS acquisition a high resolution T1weighted sagittal structural scan [magnetization prepared rapid gradient echo (MPRAGE); Repetition time (TR) $=2200 \mathrm{~ms}$; Echo time $(\mathrm{TE})=2.96 \mathrm{~ms}$; Inversion time $(\mathrm{TI})=1050 \mathrm{~ms}$; flip angle $=$ $7^{\circ}$; voxel size $=0.7 \mathrm{~mm}$ isotropic; 256 sagittal slices; Field of View $(\mathrm{FoV})=224 \mathrm{~mm}]$ was acquired for anatomical overlay of MRS voxel location.

MRS data were acquired using an ultra-short TE spin echo acquisition, the spin-echo full-intensity acquired localized (SPECIAL) sequence (60 averages; $\mathrm{TR}=4500 \mathrm{~ms} ; \mathrm{TE}=8.50 \mathrm{~ms}$ ) (Mekle et al., 2009; Xin et al., 2013). VAPOR (variable power RF pulses with optimized relaxation delays) water suppression was used (Tkác et al., 1999), and outer volume suppression was used to eliminate signal contamination from outside the MRS voxel. Shimming was performed using the Siemens product manual shim and the mean linewidth for the acquired data were 0.03 (SD $=0.006)$. An $8 \mathrm{~cm}^{3}(2 \times 2 \times 2 \mathrm{~cm})$ voxel of interest was positioned in the occipital cortex (identified using anatomical landmarks). This voxel position was selected as it has been demonstrated to provide good quality MRS spectra and as any metabolite changes resulting from exercise would be expected to be global (Mekle et al., 2009). The voxel was centered on the mid-sagittal plane with its posterior boundary positioned $6 \mathrm{~mm}$ anterior to the skull

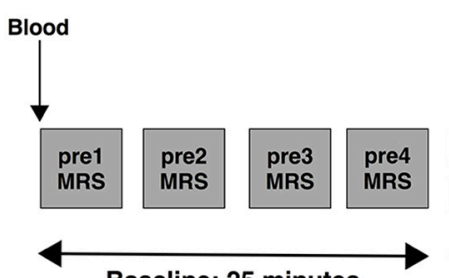

Baseline: 25 minutes

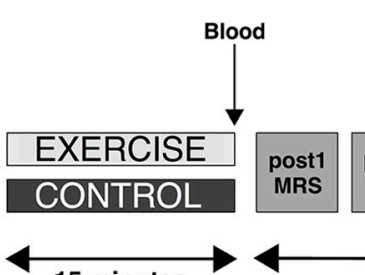

15 minutes
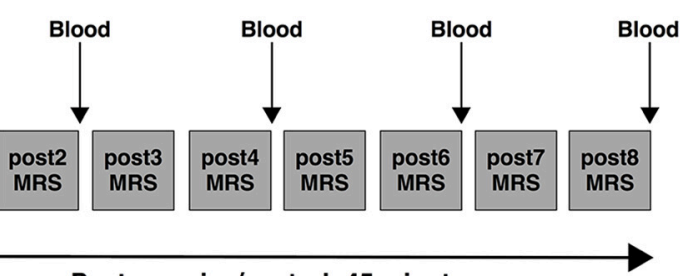

Post exercise/control: 45 minutes

FIGURE 1 | Schematic depicting the study protocol. Participants underwent two 90-min scanning sessions on two different days. On one of the days they received an exercise intervention. On the other day, they received the control intervention (quiet reading). The order of the days were randomly assigned and counterbalanced. In both sessions participants first underwent a series of baseline (pre) scans involving four repeated MRS measurements (depicted by gray squares), came out of the scanner for the intervention, and then returned to the scanner for a series of post-intervention scans (post) involving eight repeated MRS measures. Blood lactate measurements (denoted as "Blood") were taken from the big toe before the pre-scan, at the end of the intervention period, and after post-MRS measurements $2,4,6$, and 8 . 
at its point of closest proximity (Figure 2). Voxel placement was performed immediately after shimming on a rapidly acquired localiser scan prior to MRS measurement. Spectra were collected in blocks consisting of 60 acquired volumes, corresponding to a scan time of 4.5 min per block. Each scan session consisted of four pre time points and eight post time points. After the last MRS acquisition, a second T1-weighted structural scan was collected to allow registration of the post MRS voxels to those acquired at baseline.

\section{MR Data Processing}

MRS data pre-processing was conducted in Matlab and included removal of motion corrupted averages (typically only one or two were removed), and retrospective frequency and phase drift correction by spectral registration (Near et al., 2014). Spectra were quantified using LCModel (Provencher, 2001). The basis set used was generated in-house using the FID-A Simulation
Toolbox (http://github.com/CIC-methods/FID-A). Simulations consisted of an ideal spin echo sequence at $7 \mathrm{~T}$ with an echo time equal to that of our MRS acquisition ( $8.5 \mathrm{~ms})$. Only metabolites that could be quantified with Cramér-Rao lower bounds $<25 \%$ were included in the analysis. The concentrations of all the metabolites were expressed relative to total creatine (tCr; creatine + phosphocreatine) as absolute quantification of metabolites has significant uncertainties, and we did not acquire simultaneous non-suppressed water spectra, so this approach may be misleading. However, in order to eliminate the chances of changes in metabolites being driven by change in creatine, statistics for absolute values are also reported.

To determine overlap between the pre and post voxels the two structural T1 scans were linearly registered using FLIRT (FMRIB's Linear Image Registration Tool; (Jenkinson and Smith, 2001) from the FMRIB software library (FSL; Smith et al., 2004). The post voxel masks were

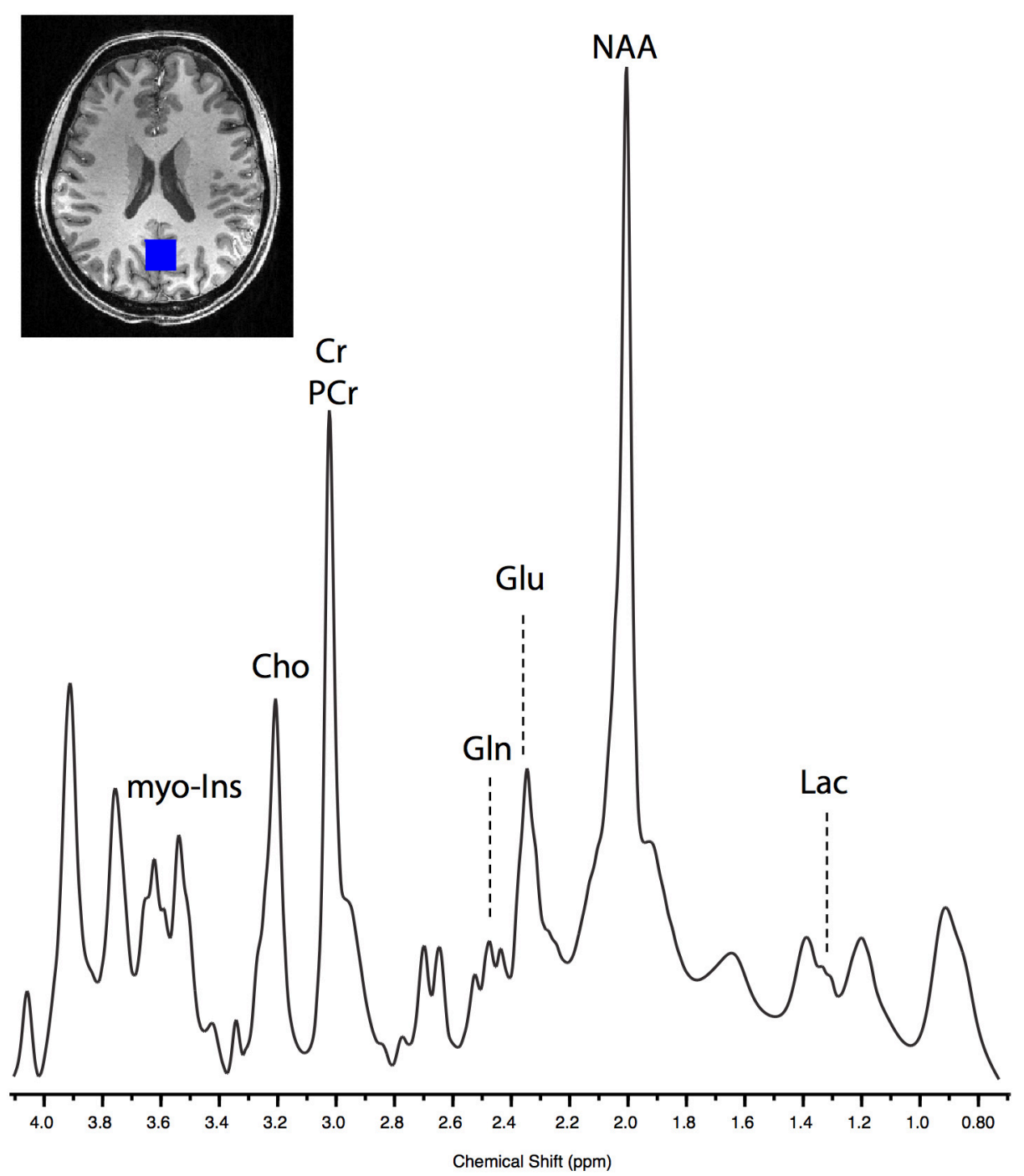

FIGURE 2 | Example of raw SPECIAL spectra acquired from one post-exercise block in a single subject. The lactate peak can be seen centered at 1.33 ppm, and glutamate (Glu) and glutamine (Gln) can be clearly separated. 
transformed into the space of the pre scan and overlap was determined using the fslmaths tool from FSL 5.1 (www.fmrib.ox.ac.uk/fsl).

\section{Statistical Analysis}

Statistical analysis was conducted on all pre and post measures of blood and of brain lactate, glutamate, and glutamine. To compare the effects of exercise vs. control, a longitudinal Linear Mixed Effects (LME) modeling approach was used, implemented in the nlme package of the $\mathrm{R}$ statistical programming language (Singer and Willett, 2003; Pinheiro et al., 2013). LME is similar to hierarchical linear regression in that explanatory variables are sequentially added and tested to determine if they explain a statistically significant amount of the variance to warrant inclusion in the final model, but has the advantage of separately modeling variance due to within and between subject factors (Singer and Willett, 2003). LME models have become a popular tool for analysing longitudinal neuroimaging data because of their flexibility to account for correlation within subjects (BernalRusiel et al., 2013; Guillaume et al., 2014). Due to potential timevarying confounds, such as scanner drift, our null model was a linear change with time regardless of intervention.

All blood lactate values were log-transformed as blood lactate has a highly skewed distribution (Foster et al., 1978). To compare baseline levels of blood lactate between sessions, paired $t$-tests were performed. To explore the relationship between metabolite concentration and PWC, correlation analysis with a standard Fisher transformation was used.

\section{RESULTS}

Subjects' mean $( \pm S D)$ weight was $68.0 \mathrm{~kg}( \pm 13.7)$ and height was $1.72 \mathrm{~m}( \pm 0.10)$. In the exercise condition, subjects exercised to an average of $87 \%( \pm 3.6)$ of their age-predicted maximal heart rate. Average maximum exercise intensity was $185 \mathrm{~W}( \pm 35)$. Average time post-intervention to the beginning of the first MRS acquisition was $32 \mathrm{~min}( \pm 10)$.

Good registration of pre and post voxels was achieved for all subjects. The pre and post voxels within individual subjects overlapped in volume by $67.1 \%( \pm 4.7)$. Consistent with previous reports, LCModel resolved the spectra of 20 different metabolites reliably (Mekle et al., 2009).

\section{Exercise and Brain Creatine Concentration}

Although not initially a metabolite of interest in the study, the metabolic nature of the paradigm deemed it necessary to examine the effect of exercise on creatine as this was the metabolite by which all others are expressed in relation to. Total creatine was successfully resolved at $3.03 \mathrm{ppm}$, with successful measurement for all time-points. Using the LME approach; the model including the post-exercise variable ( 0 or 1$)$ explained significantly more of the variance than the null model (likelihood ratio $=15.90$, $P=0.0001$ ) demonstrating that brain creatine was significantly decreased from baseline after exercise but not after the control intervention (Supplementary Figure 1). Based on this finding, all subsequent statistical analysis were conducted on both the metabolite/Cr ratio and on the absolute concentrations in order

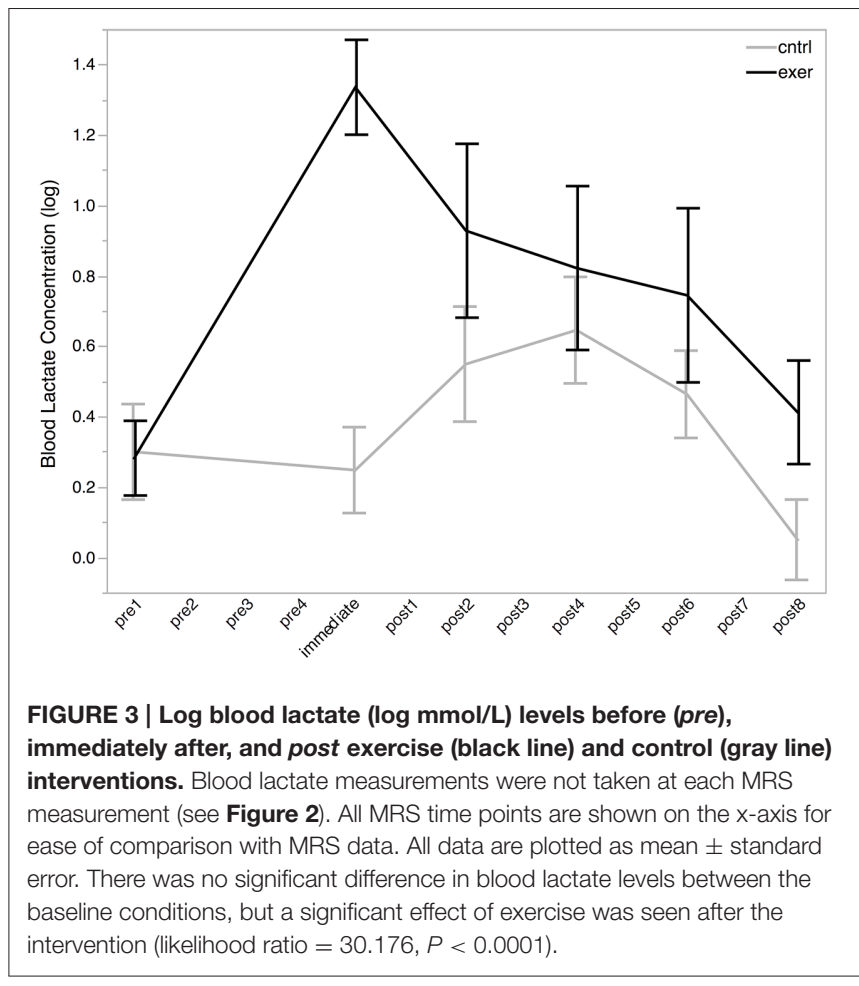

to rule out the effect of decreasing creatine on the observed patterns in lactate, glutamate, and glutamine.

\section{Exercise Increases Blood Lactate Concentration}

Blood lactate was acquired in $91 \%$ of cases. Mean blood lactate concentration was $1.4 \pm 0.6 \mathrm{mmol} / \mathrm{L}$ at baseline. Paired $t$-test revealed no significant difference between baseline blood lactate levels in the two sessions $\left[t_{(10)}=0.12, P=0.908\right]$. Following the exercise session, blood lactate levels were increased from baseline to $4.2 \pm 2.1 \mathrm{mmol} / \mathrm{L}$ (Figure 3). To test this change statistically, we used a LME approach. We constructed a model to explain changes in blood lactate across all subjects, sessions, and time points using three explanatory variables: subject ID, session, time, and a variable indicating whether the data point was collected after the exercise session ( 0 or 1 ). This model provided a significantly better fit for the variance in blood lactate than the null model which included only subject ID, session, and time (likelihood ratio $=30.176, P<0.0001$ ) demonstrating that exercise significantly increased blood lactate level. Importantly, this increase in blood lactate was maintained for at least the first two blocks of the post MRS measurements.

\section{Exercise Increases Brain Lactate Concentration}

As has been demonstrated previously (Mekle et al., 2009; Schaller et al., 2013) brain lactate could be accurately quantified at $1.33 \mathrm{ppm}$ within the SPECIAL spectra (see Figure 2 for representative spectrum). Measurement of brain lactate was successful for $78 \%$ of MRS acquisitions. Using the same LME approach described above, we found that the model including 
the post-exercise variable ( 0 or 1$)$ explained significantly more of the variance than the null model (ratio to $\mathrm{Cr}$ : likelihood ratio $=8.066, P=0.005$; absolute: likelihood ratio $=7.69$, $P=0.006)$ demonstrating that brain lactate was significantly increased from baseline after exercise but not after the control intervention (Figure 4A). In a further analysis designed to assess the correlation between brain lactate and blood lactate, log blood lactate measures were added as a covariate into the LME model. Whilst this analysis did reduce the number of data points in the model (from 286 to 121) as blood lactate was measured after every other MRS acquisition after the intervention, this model provided a significantly better fit than the same model without log blood lactate included (ratio to Cr: $\log$ likelihood $=3.839, P=0.05$; absolute: $\log$ likelihood $=6.737, P=0.0094)$. This indicates a correlation exists between blood and brain lactate measures.

\section{Glutamate and Glutamine}

Glutamate and glutamine were reliably resolved, with successful measurements at $99 \%$ of time points for glutamate and at $94 \%$ of time points for glutamine. There was no significant change in glutamate (Figure 4B) or glutamine (Figure 4C) in either session when normalized by creatine. The model including the exercise intervention was not a significantly better fit than the null model for glutamate (likelihood ratio $=1.158, P=0.281$ ) or glutamine (likelihood ratio $=1.759, P=0.185$ ), suggesting that exercise did not result in a significant change in either metabolite. However, when not normalizing by creatine, the models for both glutamate (likelihood ratio $=7.34, P=0.007$ ) and glutamine (likelihood ratio $=4.03, P=0.045$ ) showed a significantly better fit to the data when including the exercise variable.

\section{Effect of Aerobic Fitness}

It has previously been suggested that aerobic fitness modulates glutamatergic processing (Guezennec et al., 1998). In order to investigate this we first explored whether the mean baseline (pre) glutamate levels were related to the PWC150, a proxy measure of fitness. We demonstrated a significant negative correlation between baseline glutamate levels and fitness, such that fitter people had lower resting glutamate levels than less fit people $(r=-0.627, P=0.039$, Figure 5A). We then went on to see whether this relationship could also be demonstrated immediately after exercise, i.e., whether any of the inter-subject variability in glutamate after the exercise condition could be explained by subject fitness. A correlational analysis revealed that brain glutamate immediately post-exercise and PWC were significantly negatively correlated $(r=-0.726, P=0.012$; Figure 5B), such that fitter people had lower glutamate levels immediately post-exercise than less fit people. This relationship was not significantly different from that seen at baseline (Fisher's R-to-Z, $Z=0.12, P=0.9$ ).

\section{DISCUSSION}

This study investigated the effects of exercise-induced blood lactate increases on the levels of the brain metabolites lactate, glutamate, and glutamine in healthy adults. As in one previous study (Maddock et al., 2011), we have demonstrated

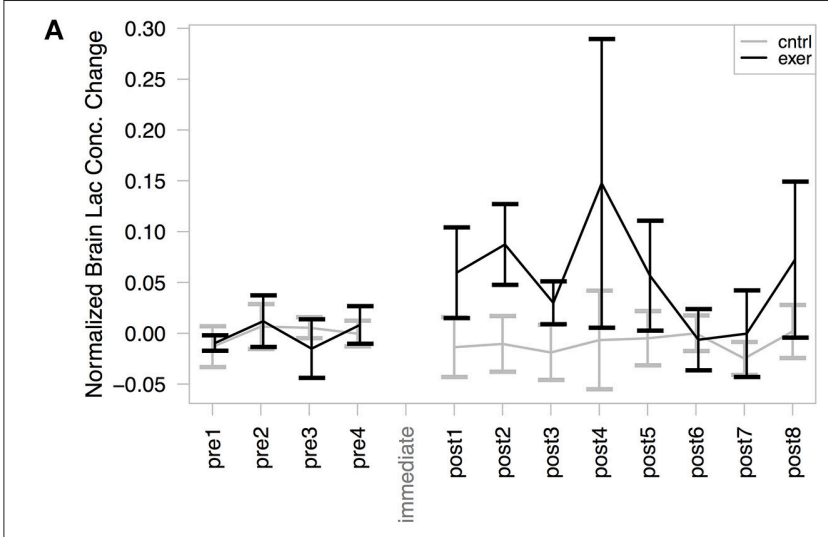

B

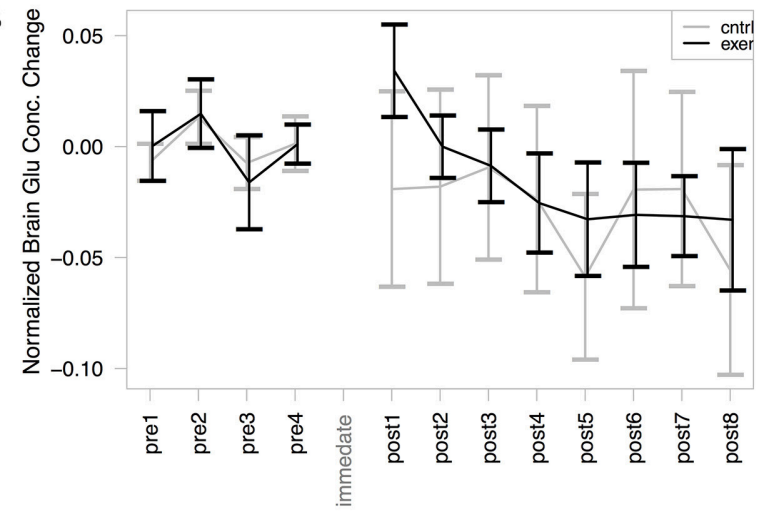

C

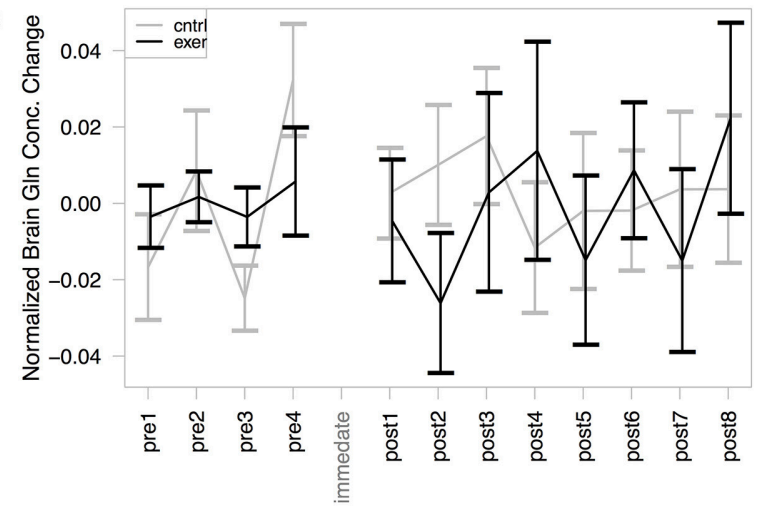

FIGURE 4 | MRS assessment of (A) Brain lactate/Cr; (B) Brain glutamate/Cr; and (C) Brain glutamine/ $\mathrm{Cr}$ change from baseline within our voxel of interest. In each case the exercise session is shown in black and the control in gray. The mean baseline concentration for each session is subtracted from the values to illustrate change. A significant effect of exercise was demonstrated in the post-exercise lactate levels, compared with those from the control session (likelihood ratio $=8.066, P=0.005$ ). No significant changes were observed in glutamate or glutamine concentration with either the exercise or the control condition.

a transient increase in brain lactate after vigorous exercise as measured through ultra-high field strength MRS. In the brain at rest, metabolite concentrations are likely to be homeostatically controlled (Balaban et al., 1986; Hochachka, 2003). Whilst significant increases in brain-derived lactate have been reported in healthy subjects during prolonged visual and motor stimulations (Prichard et al., 1991; Sappey-Marinier et al., 


\section{A}

\section{No Exercise}

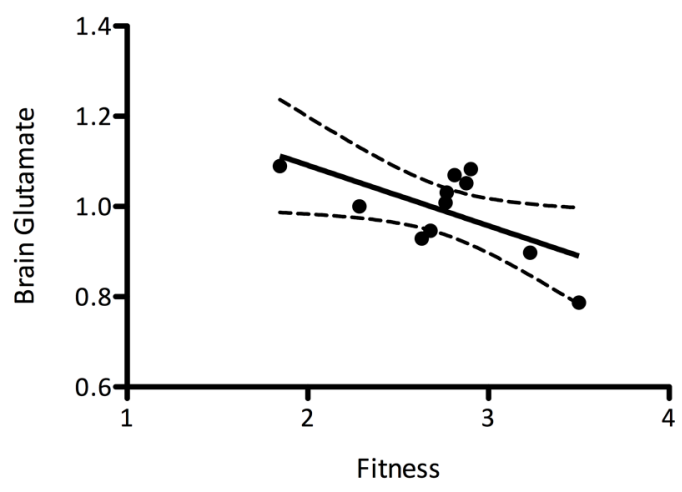

B

Immediately post-exercise

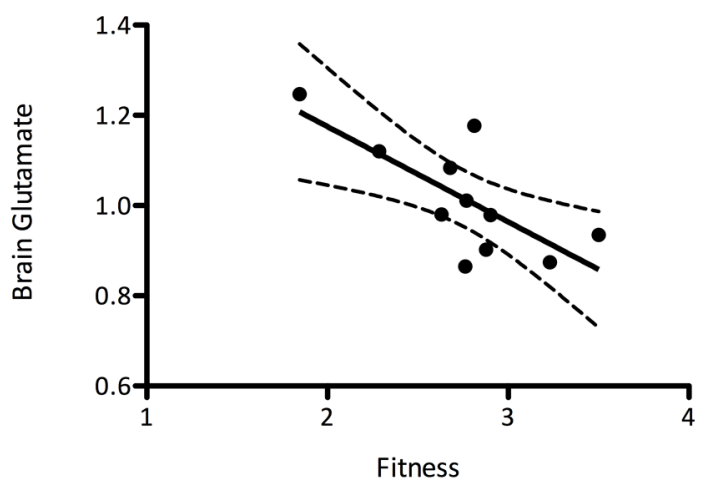

FIGURE 5 | (A) A significant negative correlation between brain glutamate levels at rest and fitness $(r=-0.627, P=0.039)$, such that fitter people had lower resting glutamate levels. (B) This correlation is also present immediately after exercise $(r=-0.726, P=0.012)$. The relationship between brain glutamate levels and fitness are not significantly altered after exercise (Fisher's R-to-Z, $Z=0.12, P=0.9$ ).

1992; Mangia et al., 2007; Schaller et al., 2014), such increases are transient and return to baseline within minutes. Therefore, there is no reason to expect an increase in brain tissue lactate between the pre and post scan in the current study, when neither acquisition required any task performance during data collection. We therefore believe our results support the hypothesis that lactate is taken up by the brain when available in high abundance in the plasma. However, unlike the previous study, we were able to resolve MRS peaks for both glutamine and glutamate. We did not observe any significant change in either amino acid following exercise, although we did observe a significant negative correlation between a proxy measure of aerobic fitness and glutamate.

The uptake and oxidization of lactate by the brain, heart, and muscle when available in the blood stream is not a newly discovered phenomenon, with a vast literature supporting its occurrence (Brooks, 1985, 2009; Schurr et al., 1997; Ide et al., 2000; van Hall et al., 2009; Bouzat et al., 2014). Here, for the first time, we present data from an ultra-high field
MR study of brain metabolite concentration changes following exercise. This is an important advance, since MRS at $7 \mathrm{~T}$ allows for the accurate, non-invasive quantification of a wide range of metabolites, most pertinently here including lactate, glutamate, and glutamine. Although proton MRS cannot provide information on cerebral flux of metabolites between cells, or on cerebral compartmentalization, it can provide valuable in vivo information about brain metabolism.

Interestingly, we also observed a significant decrease in brain creatine following exercise. This is an important finding given the prevalence in the literature to ratio metabolites to the usually stable metabolite creatine. Further studies interested in the fate of brain metabolites following exercise should consider reporting absolute concentrations based on normalization to unsuppressed water spectrum.

\section{The Metabolic Fate of Peripherally-derived Brain Lactate in the Brain}

A potential limitation with this type of experiment is that it is difficult to determine whether a change in lactate is due to increased brain uptake of plasma lactate or increases in locally produced lactate as a result of increased cerebral metabolism. However, results from our statistical analysis comparing brain lactate change were strengthened when blood lactate was added to the model, indicating a correlation exists between the brain and blood measures. This result, coupled with the absence of a change in glutamate and the temporal delay in acquiring our post-exercise measures, would be supportive of the increased uptake of plasma lactate. Thus, we believe our results support previous findings that lactate is taken up into the brain when it is in high supply in the bloodstream (Quistorff et al., 2008; van Hall et al., 2009). It has previously been shown that in the absence of exercise, all lactate taken up by the brain is oxidized, accounting for approximately $10 \%$ of cerebral energy requirements (Boumezbeur et al., 2010). During exercise, lactate accounts for $33 \%$ cerebral energy requirements (Overgaard et al., 2012 ), and may provide up to $66 \%$ of energy requirements when found in high concentration in the blood (Boumezbeur et al., 2010). Studies have suggested that the majority of plasma lactate is metabolized by the neurons (Boumezbeur et al., 2010). Whilst an interesting finding, and potentially in line with the hypothesis supporting a preferential use of lactate as a fuel source in exercising human subjects (Quistorff et al., 2008; van Hall, 2010), the MRS measurement is a total concentration and cannot dissociate between lactate within astrocytes and neurons; thus, we cannot conclude whether it is preferred to glucose following exercise. In addition, direct quantification of cerebral glucose levels was not possible with the MRS method used here and, therefore, we cannot comment on any changes in global brain glucose uptake.

In line with our hypothesis, but in contrast to a previous study in humans (Maddock et al., 2011), we did not observe any significant change in glutamate or glutamine following vigorous exercise, when the metabolites were normalized to creatine. When "absolute" quantification was performed, a significant decrease in both glutamate and glutamine after exercise was observed. As discussed earlier, presenting metabolites as a ratio 
to creatine is common practice as it is taken to provide a stable, simultaneously acquired reference metabolite, but here we see a significant decrease in creatine following exercise. In light of this, we also explored the changes in the absolute quantification of glutamate and glutamine, although we acknowledge there are uncertainties with this approach. With this analysis, a significant decrease in glutamate and glutamine was observed, in contrast to the increase observed by Maddock et al. (2011). It is not possible, therefore, given the discrepancy between these methods, to be confident as to whether there is a true decrease in glutamate and glutamine after exercise, but certainly our data do not support an increase in these metabolites, as previously observed.

Conceptually, glutamate concentration might be expected to increase in proportion to overall neuronal activity. During exercise, large global increases in cerebral metabolism are seen (van Praag, 2009). Evidence from the literature would suggest that non-oxidized carbohydrates, such as lactate, are only metabolized into glutamate when there is an increase in "brain work" demanding more glutamatergic neural activation, and an adequate source of ammonia (Hertz and Dienel, 2004). Therefore, measurements of glutamate, and lactate, taken during exercise may be expected to be elevated as a result of local production. However, our MRS measures were acquired some time after the cessation of exercise, and it is therefore unlikely that we would observe a heightened state of brain activity, and hence an increase in glutamate (MacIntosh et al., 2014).

Nevertheless, glutamate and glutamine changes may be observed in the presence of high brain lactate in some animal models (Waagepetersen et al., 1998; Hertz and Fillenz, 1999). For example, lactate may be partially oxidized in the TCA cycle allowing for glutamate to be oxidized to alpha-ketoglutarate, or for increased glutamate-glutamine cycling in the astrocytes in response to neural activity. However, these mechanisms largely depend on an increase in brain-derived lactate generated by the astrocytes in response to neural activation, and are not involved in response to an increase in plasma lactate. Studies of peripherally derived lactate uptake into the brain following either infusion of lactate or following exercise have reported virtually all ( $\sim 87 \%$ ) lactate taken up is recovered as $\mathrm{CO}_{2}$ (Volianitis et al., 2008; van Hall et al., 2009), suggesting that there is little carbon being exchanged from lactate to other metabolites (van Hall et al., 2009).

\section{Relationship Between Glutamate and Fitness}

Whilst previous studies have reported differences in the NAA/Cr ratio in frontal gray matter and Cho/Cr ratios in occipitoparietal gray matter between aerobically trained and sedentary adults (Gonzales et al., 2013) and a correlation between aerobic fitness and the frontal NAA/Cr ratio (Erickson et al., 2012), currently no studies have reported differences in $\mathrm{Glu} / \mathrm{Cr}$ ratios between trained and sedentary individuals (Gonzales et al., 2013), potentially due to the methodological difficulties in separating glutamate from glutamine.

The negative relationship we observed between fitness and glutamate may provide some support to the beneficial effects of regular exercise participation on brain health. For example, in rat models, exercise training results in decreased glutamatergic activity in response to oxygen and glucose deprivation in hippocampal slices (Mourão et al., 2014), and lowers the glutamatergic input to the rostro ventrolateral medulla (Zha et al., 2013), a phenomenon associated with lowering blood pressure. Therefore, our finding of lower glutamate levels in fitter subjects could be related to the neuro-protective benefits of regular physical exercise. It should be noted however, that the exercise paradigm was not designed as an exercise test and thus a subsequent proxy measure of fitness (PWC) was inferred from the available data. In light of the interesting relationship between PWC and glutamate, an established test of aerobic fitness or lactate threshold may provide a more comprehensive metric for examining the relationship between fitness and brain activity. It should also be noted that, whilst an interesting trend, further study of a population with a range of aerobic fitness levels would be necessary in order to elaborate on any proposed relation between fitness and resting brain glutamate levels.

\section{Limitations}

There are some limitations of the methods used in this study. The MRS technique allows for non-invasive measurement of neurochemicals in vivo, making it ideal for longitudinal studies in human populations. However, it has inherently lower signal-tonoise than more direct measures. MRS allows for measurement from a single ROI. The voxel in the visual cortex was selected based on the assumption that changes in brain lactate as taken up from the blood would be global and not regionally specific, and lactate peaks have previously been successfully resolved at $7 \mathrm{~T}$ in the visual cortex (Mangia et al., 2007; Mekle et al., 2009). However, this method required the voxel to be manually selected. Whilst every care was taken to ensure consistency, variations may have influenced the relative change in metabolites, though the reproducibility across the baseline and post-intervention time points suggests this is unlikely.

We only acquired an unsuppressed water spectrum at the beginning of the baseline scan and the post-intervention scan. We do not feel that this is an appropriate reference for the later post-exercise scans as hydration, as well as other physiological variables, might change. Secondly, there are significant uncertainties in calculating the "absolute" concentrations (highlighted in chapter 10 of the LCModel manual). Thirdly, we wished to extend the findings of Maddock et al. (2011), and so wished our results to be as comparable to that study, which used a creatine reference, as possible. However, given the discrepancy between our two quantification methods, future studies should include the appropriate scans to allow accurate absolute quantification to be performed.

It should also be acknowledged that the ROI approach gives us access to data from a specific region of the brain, which includes a number of constituents including brain tissue, CSF, and blood, and that in this voxel, with its proximity to the draining veins, the venous component might potentially be significant. However, we would argue strongly that the MRS measures we acquired here are not sensitive to signal from the blood within the voxel for a number of reasons. Whilst the initial excitation pulse will excite all the components of the ROI, the TE means that the 
vast majority of the blood that was in the voxel when the RF was applied will no longer be present, and therefore will not contribute to the signal acquired. This will be true of any arterial, arteriolar, and capillary supply within the voxel, where flow will be fast enough to remove a significant portion of the blood. Potentially, however, venous drainage in the sagittal sinus might be more sluggish than this. We were therefore careful to place the voxel sufficiently anterior of the sinuses to avoid this confound.

Whilst MRS offers an in vivo and non-invasive method for measuring brain metabolites, this "snapshot" measurement approach means only total, static measures are possible and measurement of flux is not. For information on metabolic flux, measures such as ${ }^{13} \mathrm{C}$ MRS would need to be employed.

Lastly, the time-interval between the ending of the exercise session and the start of the first post-exercise scan was variable. It should be noted however that blood lactate levels were still elevated from baseline during first post-exercise MRS acquisition maintaining the integrity of the protocol to examine peripherally derived lactate. Further study of the changes in brain lactate and glutamate immediately after exercise may shed further light on brain metabolism and activity changes in the immediate recovery period.

\section{CONCLUSIONS}

We observed a transient increase in brain lactate following vigorous exercise when blood lactate was elevated. We were also able to successfully measure brain glutamate and glutamine, and in line with our hypothesis, though contrary to previous findings (Maddock et al., 2011), we did not observe any change in either as a result of exercise. We did observe a negative correlation between brain glutamate and fitness, both after exercise and at

\section{REFERENCES}

Balaban, R. S., Kantor, H. L., Katz, L. A., and Briggs, R. W. (1986). Relation between work and phosphate metabolite in the in vivo paced mammalian heart. Science 232, 1121-1123. doi: 10.1126/science.3704638

Bangsbo, J., Graham, T., Johansen, L., and Saltin, B. (1994). Muscle lactate metabolism in recovery from intense exhaustive exercise: impact of light exercise. J. Appl. Physiol. 77, 1890-1895.

Bernal-Rusiel, J. L., Greve, D. N., Reuter, M., Fischl, B., and Sabuncu, M. R. (2013). Statistical analysis of longitudinal neuroimage data with linear mixed effects models. Neuroimage 66, 249-260. doi: 10.1016/j.neuroimage.2012.10.065

Boumezbeur, F., Petersen, K. F., Cline, G. W., Mason, G. F., Behar, K. L., Shulman, G. I., et al. (2010). The contribution of blood lactate to brain energy metabolism in humans measured by dynamic $13 \mathrm{C}$ nuclear magnetic resonance spectroscopy. J. Neurosci. 30, 13983-13991. doi: 10.1523/JNEUROSCI.204010.2010

Bouzat, P., Sala, N., Suys, T., Zerlauth, J.B., Marques-Vidal, P., Feihl, F., et al. (2014). Cerebral metabolic effects of exogenous lactate supplementation on the injured human brain. Intensive Care Med. 40, 412-421. doi: 10.1007/s00134013-3203-6

Bouzier-Sore, A.K., Voisin, P., Canioni, P., Magistretti, P. J., and Pellerin, L. (2003). Lactate is a preferential oxidative energy substrate over glucose for neurons in culture. J. Cereb. Blood Flow Metab. 23, 1298-1306. doi: 10.1097/01.WCB.0000091761.61714.25

Brooks, G. A. (2009). Cell-cell and intracellular lactate shuttles. J. Physiol. 587, 5591-5600. doi: 10.1113/jphysiol.2009.178350 rest. These results provide support for the use of high-field MRS for measuring post-exercise brain metabolites.

\section{FUNDING}

The work was supported by the National Institute for Health Research (NIHR) Oxford Biomedical Research Center based at Oxford University Hospitals Trust, Oxford University. The views expressed are those of the author(s) and not necessarily those of the NHS, the NIHR, or the Department of Health. AT is supported by the NIMH Intramural Research Program based in Bethesda, MD. HJ is a Wellcome Trust Senior Research Fellow. CS holds a Sir Henry Dale Fellowship jointly funded by the Wellcome Trust and the Royal Society (Grant Number 102584/Z/13/Z).

\section{ACKNOWLEDGMENTS}

The authors are grateful to Anderson Winkler for statistical support, Isidro Hidalgo Arellano for assistance with $\mathrm{R}$ visualization, and to Martyn Morris for expert assistance.

\section{SUPPLEMENTARY MATERIAL}

The Supplementary Material for this article can be found online at: http://journal.frontiersin.org/article/10.3389/fphys. 2015.00351

Supplementary Figure 1 | MRS assessment of absolute creatine (Cr + Pcr) within the voxel of interest. Mean baseline concentration for each session is subtracted from the values to illustrate change. Exercise session is shown in black and the control in gray. A significant effect of exercise was demonstrated in the post-exercise creating levels, compared with those from the control session (likelihood ratio $=15.90, P=0.0001$ ).

Brooks, G. A. (1985). The lactate shuttle during exercise and recovery. Med. Sci. Sport Exerc. 18, 360-368. doi: 10.1249/00005768-198606000-00019

Brooks, G. A., and Martin, N. A. (2015). Cerebral metabolism following traumatic brain injury: new discoveries with implications for treatment. Front. Neurosci. 8:408. doi: $10.3389 /$ fnins. 2014.00408

Campbell, P. T., Katzmarzyk, P. T., Malina, R. M., Rao, D. C., Pérusse, L., and Bouchard, C. (2001). Prediction of physical activity and physical work capacity (PWC150) in young adulthood from childhood and adolescence with consideration of parental measures. Am. J. Hum. Biol. 13, 190-196. doi: 10.1002/1520-6300(200102/03)13:2\&lt;190::AID-AJHB1028\&gt; 3.0.CO;2-N

Cerdán, S., Rodrigues, T. B., Sierra, A., Benito, M., Fonseca, L. L., Fonseca, C. P., et al. (2006). The redox switch/redox coupling hypothesis. Neurochem. Int. 48, 523-530. doi: 10.1016/j.neuint.2005.12.036

Chowdhury, G. M., Jiang, L., Rothman, D. L., and Behar, K. L. (2014). The contribution of ketone bodies to basal and activity-dependent neuronal oxidation in vivo. J. Cereb. Blood Flow Metab. 34, 1233-1242. doi: $10.1038 /$ jcbfm. 2014.77

Cruz, F., Villalba, M., García-Espinosa, M. A., Ballesteros, P., Bogónez, E., Satrústegui, J., et al. (2001). Intracellular compartmentation of pyruvate in primary cultures of cortical neurons as detected by (13)C NMR spectroscopy with multiple (13)C labels. J. Neurosci. Res. 66, 771-781. doi: 10.1002/jnr. 10048

Dalsgaard, M. K., Ide, K., Cai, Y., Quistorff, B., and Secher, N. H. (2002). The intent to exercise influences the cerebral $\mathrm{O} 2 /$ carbohydrate uptake ratio in humans. J. Physiol. 540, 681-689. doi: 10.1113/jphysiol.2001.013062 
Dalsgaard, M. K., Quistorff, B., Danielsen, E. R., Selmer, C., Vogelsang, T., and Secher, N. H. (2004). A reduced cerebral metabolic ratio in exercise reflects metabolism and not accumulation of lactate within the human brain. J. Physiol. 554, 571-578. doi: 10.1113/jphysiol.2003.055053

Dienel, G. A. (2012). Brain lactate metabolism: the discoveries and the controversies. J. Cereb. Blood Flow Metab. 32, 1107-1138. doi: 10.1038/jcbfm.2011.175

Erickson, K. I., Weinstein, A. M., Sutton, B. P., Prakash, R. S., Voss, M. W., Chaddock, L., et al. (2012). Beyond vascularization: aerobic fitness is associated with $\mathrm{N}$-acetylaspartate and working memory. Brain Behav. 2, 32-41. doi: $10.1002 /$ brb3.30

Forsyth, J. J., and Farrally, M. R. (2000). A comparison of lactate concentration in plasma collected from the toe, ear, and fingertip after a simulated rowing exercise. Br. J. Sport Med. 34, 35-38. doi: 10.1136/bjsm.34.1.35

Foster, K. J., Alberti, K. G., Hinks, L., Lloyd, B., Postle, A., Smythe, P., et al. (1978). Blood intermediary metabolite and insulin concentrations after an overnight fast: reference ranges for adults, and interrelations. Clin. Chem. 24, 1568-1572.

Fox, P. T., Raichle, M. E., Mintun, M. A., and Dence, C. (1988). Nonoxidative glucose consumption during focal physiologic neural activity. Science 241, 462-464. doi: 10.1126/science.3260686

Garland, S. W., and Atkinson, G. (2008). Effect of blood lactate sample site and test protocol on training zone prescription in rowing. Int. J. Sport Physiol. Perform. 3, 347-358.

Glenn, T. C., Martin, N. A., Horning, M. A., McArthur, D. L., Hovda, D. A., Vespa, P., et al. (2015). Lactate: brain fuel in human traumatic brain injury. A comparison to normal healthy control subjects. J. Neurotrauma 32, 820-832. doi: $10.1089 /$ neu.2014.3483

Gonzales, M. M., Tarumi, T., Kaur, S., Nualnim, N., Fallow, B. A., Pyron, M., et al. (2013). Aerobic fitness and the brain: increased $\mathrm{N}$-acetyl-aspartate and choline concentrations in endurance-trained middle-aged adults. Brain Topogr. 26, 126-134. doi: 10.1007/s10548-012-0248-8

Guezennec, C. Y., Abdelmalki, A., Merino, D., Bigard, X., Berthelot, M., Pierard, C., et al. (1998). Effects of prolonged exercise on brain ammonia and amino acids. Physiol. Biochem. 19, 323-327. doi: 10.1055/s-2007-971925

Guillaume, B., Hua, X., Thompson, P. M., Waldorp, L., and Nichols, T. E. (2014). Fast and accurate modelling of longitudinal and repeated measures neuroimaging data. Neuroimage 94, 287-302. doi: 10.1016/j.neuroimage.2014.03.029

Hertz, L., and Dienel, G. A. (2004). Lactate transport and transporters: general principles and functional roles in brain cells. J. Neurosci. Res. 79, 11-18. doi: 10.1002/jnr.20294

Hertz, L., and Fillenz, M. (1999). Does the "mystery of the extra glucose" during CNS activation reflect glutamate synthesis? Neurochem. Int. 34, 71-75. doi: 10.1016/S0197-0186(98)00071-0

Hertz, L., Gibbs, M. E., and Dienel, G. A., (2014). Fluxes of lactate into, from, and among gap junction-coupled astrocytes and their interaction with noradrenaline. Front. Neurosci. 8:261. doi: 10.3389/fnins.2014.00261

Hochachka, P. W. (2003). Intracellular convection, homeostasis and metabolic regulation. J. Exp. Biol. 206, 2001-2009. doi: 10.1242/jeb.00402

Ide, K., Schmalbruch, I. K., Quistorff, B., Horn, A., and Secher, N. H. (2000). Lactate, glucose and $\mathrm{O} 2$ uptake in human brain during recovery from maximal exercise. J. Physiol. 522(Pt 1), 159-164. doi: 10.1111/j.1469-7793.2000.t01-200159.xm

Jenkinson, M., and Smith, S. (2001). A global optimisation method for robust affine registration of brain images. Med. Image Anal. 5, 143-156. doi: 10.1016/S13618415(01)00036-6

MacIntosh, B. J., Crane, D. E., Sage, M. D., Rajab, A. S., Donahue, M. J., McIlroy, W. E., et al. (2014). Impact of a single bout of aerobic exercise on regional brain perfusion and activation responses in healthy young adults. PLOS ONE 9:e85163. doi: 10.1371/journal.pone.0085163

Maddock, R. J., Casazza, G. A., Buonocore, M. H., and Tanase, C. (2011). Vigorous exercise increases brain lactate and Glx (glutamate+glutamine): a dynamic $1 \mathrm{H}-$ MRS study. Neuroimage 57, 1324-1330. doi: 10.1016/j.neuroimage.2011.05.048

Magistretti, P. J., and Pellerin, L. (1999). Astrocytes couple synaptic activity to glucose utilization in the brain. News Physiol. Sci. 14, 177-182.

Mangia, S., Tkác, I., Gruetter, R., Van de Moortele, P.F., Maraviglia, B., and Uğurbil, K. (2007). Sustained neuronal activation raises oxidative metabolism to a new steady-state level: evidence from $1 \mathrm{H}$ NMR spectroscopy in the human visual cortex. J. Cereb. Blood Flow Metab. 27, 1055-1063. doi: $10.1038 /$ sj.jcbfm.9600401

Massucci, F. A., DiNuzzo, M., Giove, F., Maraviglia, B., Castillo, I. P., Marinari, E., et al. (2013). Energy metabolism and glutamate-glutamine cycle in the brain: a stoichiometric modeling perspective. BMC Syst. Biol. 7:103. doi: 10.1186/17520509-7-103

Mekle, R., Mlynárik, V., Gambarota, G., Hergt, M., Krueger, G., and Gruetter, R. (2009). MR spectroscopy of the human brain with enhanced signal intensity at ultrashort echo times on a clinical platform at 3T and 7T. Magn. Reson. Med. 61, 1279-1285. doi: 10.1002/mrm.21961

Mourão, F. A., Leite, H. R., de Carvalho, L. E., Ferreira E Vieira, T. H., Pinto, M. C., de Castro Medeiros, D., et al. (2014). Neuroprotective effect of exercise in rat hippocampal slices submitted to in vitro ischemia is promoted by decrease of glutamate release and pro-apoptotic markers. J. Neurochem. 131, 65-73. doi: 10.1111/jnc. 12786

Near, J., Edden, R., Evans, C. J., Paquin, R., Harris, A., and Jezzard, P. (2014). Frequency and phase drift correction of magnetic resonance spectroscopy data by spectral registration in the time domain. Magn. Reson. Med. 50, 44-50. doi: $10.1002 / \mathrm{mrm} .25094$

Nybo, L., Møller, K., Pedersen, B. K., Nielsen, B., and Secher, N. H. (2003). Association between fatigue and failure to preserve cerebral energy turnover during prolonged exercise. Acta Physiol. Scand. 179, 67-74. doi: 10.1046/j.1365201X.2003.01175.x

Overgaard, M., Rasmussen, P., Bohm, A. M., Seifert, T., Brassard, P., Zaar, M., et al. (2012). Hypoxia and exercise provoke both lactate release and lactate oxidation by the human brain. FASEB J. 26, 3012-3020. doi: 10.1096/fj.11191999

Pellerin, L., and Magistretti, P. J. (1994). Glutamate uptake into astrocytes stimulates aerobic glycolysis: a mechanism coupling neuronal activity to glucose utilization. Proc. Natl. Acad. Sci. U.S.A. 91, 10625-10629. doi: 10.1073/pnas.91.22.10625

Pellerin, L., and Magistretti, P. J. (2012). Sweet sixteen for ANLS. J. Cereb. Blood Flow Metab. 32, 1152-1166. doi: 10.1038/jcbfm.2011.149

Pinheiro, J., Bates, D., DebRoy, S., Sarkar, D., and Team, R. C. (2013). nlme: Linear and Nonlinear Mixed Effects Models. Available online at: http://cran.r-project. org/package $=$ nlme

Prichard, J., Rothmans, D., Novotny, E., Petroff, O., Kuwabara, T., Avisonf, M., et al. (1991). Lactate rise detected by 1 H NMR in human visual cortex during physiologic stimulation. Proc. Natl. Acad. Sci. U.S.A. 88, 5829-5831.

Provencher, S. W. (2001). Automatic quantitation of localized in vivo $1 \mathrm{H}$ spectra with LCModel. NMR Biomed. 14, 260-264. doi: 10.1002/nbm.698

Quistorff, B., Secher, N. H., and Van Lieshout, J. J. (2008). Lactate fuels the human brain during exercise. FASEB J. 22, 3443-3449. doi: 10.1096/fj.08-106104

Sappey-Marinier, D., Calabrese, G., Fein, G., Hugg, J. W., Biggins, C., and Weiner, M. W. (1992). Effect of photic stimulation on human visual cortex lactate and phosphates using $1 \mathrm{H}$ and $31 \mathrm{P}$ magnetic resonance spectroscopy. J. Cereb. Blood Flow Metab. 12, 584-592. doi: 10.1038/jcbfm.1992.82

Schaller, B., Mekle, R., Xin, L., Kunz, N., and Gruetter, R. (2013). Net increase of lactate and glutamate concentration in activated human visual cortex detected with magnetic resonance spectroscopy at 7 tesla. J. Neurosci. Res. 91, 1076-1083. doi: $10.1002 /$ jnr.23194

Schaller, B., Xin, L., O’Brien, K., Magill, A. W., and Gruetter, R. (2014). Are glutamate and lactate increases ubiquitous to physiological activation? A (1)H functional MR spectroscopy study during motor activation in human brain at 7Tesla. Neuroimage 93(Pt 1), 138-145. doi: 10.1016/j.neuroimage.2014. 02.016

Schurr, A. (2008). Lactate: a major and crucial player in normal function of both muscle and brain. J. Physiol. 586, 2665-2666. doi: 10.1113/jphysiol.2008. 155416

Schurr, A. (2014). Cerebral glycolysis: a century of persistent misunderstanding and misconception. Front. Neurosci. 8:360. doi: 10.3389/fnins.2014.00360

Schurr, A., Payne, R. S., Miller, J. J., and Rigor, B. M. (1997). Brain lactate is an obligatory aerobic energy substrate for functional recovery after hypoxia: further in vitro validation. J. Neurochem. 69, 423-426.

Schurr, A., West, C. A., and Rigor, B. M. (1988). Lactate-supported synaptic function in the rat hippocampal slice preparation. Science 240, 1326-1328.

Sharma, P., Karian, J., Sharma, S., Liu, S., and Mongan, P. D. (2003). Pyruvate ameliorates post ischemic injury of rat astrocytes and protects 
them against PARP mediated cell death. Brain Res. 992, 104-113. doi: 10.1016/j.brainres.2003.08.043

Shen, J., and Rothman, D. L. (2002). Magnetic resonance spectroscopic approaches to studying neuronal: glial interactions. Biol Psychiatry 52, 694-700. doi: 10.1016/S0006-3223(02)01502-0

Sibson, N. R., Dhankhar, A., Mason, G. F., Rothman, D. L., Behar, K. L., and Shulman, R. G. (1998). Stoichiometric coupling of brain glucose metabolism and glutamatergic neuronal activity. Proc. Natl. Acad. Sci. U.S.A. 95, 316-321.

Singer, J. D., and Willett, J. B. (2003). Applied Longitudinal Data Analysis: Modeling Change and Event Occurrence. New York, NY: Oxford University Press. doi: 10.1093/acprof:oso/9780195152968.001.0001

Smith, S. M., Jenkinson, M., Woolrich, M. W., Beckmann, C. F., Behrens, T. E., Johansen-Berg, H., et al. (2004). Advances in functional and structural MR image analysis and implementation as FSL. Neuroimage 23(Suppl. 1), S208-S219. doi: 10.1016/j.neuroimage.2004.07.051

Tkác, I., Starcuk, Z., Choi, I. Y., and Gruetter, R. (1999). In vivo 1H NMR spectroscopy of rat brain at $1 \mathrm{~ms}$ echo time. Magn. Reson. Med. 41, 649-656.

van Hall, G. (2010). Lactate kinetics in human tissues at rest and during exercise. Acta Physiol. (Oxf). 199, 499-508. doi: 10.1111/j.1748-1716.2010. 02122.x

van Hall, G., Strømstad, M., Rasmussen, P., Jans, O., Zaar, M., Gam, C., et al. (2009). Blood lactate is an important energy source for the human brain. J. Cereb. Blood Flow Metab. 29, 1121-1129. doi: 10.1038/jcbfm. 2009.35

van Praag, H. (2009). Exercise and the brain: something to chew on. Trends Neurosci. 32, 283-290. doi: 10.1016/j.tins.2008.12.007

Volianitis, S., Rasmussen, P., Seifert, T., Nielsen, H. B., and Secher, N. H. (2011). Plasma $\mathrm{pH}$ does not influence the cerebral metabolic ratio during maximal whole body exercise. J. Physiol. 589, 423-429. doi: 10.1113/jphysiol.2010. 195636
Volianitis, S., Fabricius-Bjerre, A., Overgaard, A., Strømstad, M., Bjarrum, M. Carlson, C., et al. (2008). The cerebral metabolic ratio is not affected by oxygen availability during maximal exercise in humans. J. Physiol. 586, 107-112. doi: 10.1113/jphysiol.2007.142273

Waagepetersen, H. S., Bakken, I. J., Larsson, O. M., Sonnewald, U., and Schousboe, A. (1998). Comparison of lactate and glucose metabolism in cultured neocortical neurons and astrocytes using 13C-NMR spectroscopy. Dev. Neurosci. 20, 310-320.

Xin, L., Schaller, B., Mlynarik, V., Lu, H., and Gruetter, R. (2013). Proton T1 relaxation times of metabolites in human occipital white and gray matter at 7 T. Magn. Reson. Med. 69, 931-936. doi: 10.1002/mrm.24352

Yang, J., Ruchti, E., Petit, J. M., Jourdain, P., Grenningloh, G., Allaman, I., et al. (2014). Lactate promotes plasticity gene expression by potentiating NMDA signaling in neurons. Proc. Natl. Acad. Sci. U.S.A. 111, 12228-12233. doi: 10.1073/pnas.1322912111

Zha, Y. P., Wang, Y. K., Deng, Y., Zhang, R. W., Tan, X., Yuan, W. J., et al. (2013). Exercise training lowers the enhanced tonically active glutamatergic input to the rostral ventrolateral medulla in hypertensive rats. CNS Neurosci. Ther. 19, 244-251. doi: 10.1111/cns.12065

Conflict of Interest Statement: The authors declare that the research was conducted in the absence of any commercial or financial relationships that could be construed as a potential conflict of interest.

Copyright (C) 2015 Dennis, Thomas, Rawlings, Near, Nichols, Clare, Johansen-Berg and Stagg. This is an open-access article distributed under the terms of the Creative Commons Attribution License (CC BY). The use, distribution or reproduction in other forums is permitted, provided the original author(s) or licensor are credited and that the original publication in this journal is cited, in accordance with accepted academic practice. No use, distribution or reproduction is permitted which does not comply with these terms. 